\title{
Prevalence of mood, panic and eating disorders in obese patients referred to bariatric surgery: patterns of comorbidity and relationship with body mass index
}

\author{
Margherita Barbuti ${ }^{1}$. Giulio E. Brancati ${ }^{1}$ - Alba Calderone ${ }^{2} \cdot$ Paola Fierabracci $^{2}$ - Guido Salvetti ${ }^{2}$. Francesco Weiss ${ }^{1}$. \\ Giulia Carignani ${ }^{1} \cdot$ Ferruccio Santini $^{2} \cdot$ Giulio Perugi $^{1}{ }^{10}$
}

Received: 13 March 2021 / Accepted: 31 May 2021 / Published online: 16 June 2021

(c) The Author(s) 2021

\begin{abstract}
Purpose We aimed at investigating the lifetime prevalence of mood, eating and panic disorders in a large sample of obese patients referred to bariatric surgery. We also explored the patterns of psychiatric comorbidity and their relationship with Body Mass Index (BMI).

Methods The sample was composed of patients consecutively referred for pre-surgical evaluation to the Obesity Center of Pisa University Hospital between January 2004 and November 2016. Clinical charts were retrieved and examined to obtain sociodemographic information, anthropometric variables and lifetime psychiatric diagnoses according to DSM-IV criteria. Results A total of 871 patients were included in the study; $72 \%$ were females, and most patients had BMI $\geq 40 \mathrm{~kg} / \mathrm{m}^{2}(81 \%)$. Overall, 55\% of the patients were diagnosed with at least one lifetime psychiatric disorder. Binge eating disorder (27.6\%), major depressive disorder (16\%), bipolar disorder type $2(15.5 \%)$, and panic disorder (16\%) were the most common psychiatric diagnoses. Mood disorders showed associations with panic disorder $\left(\mathrm{OR}=2.75,95 \% \mathrm{CI}=1.90-3.99, \chi^{2}=41.85\right.$, $p=0.000)$ and eating disorders $\left(\mathrm{OR}=2.17,95 \% \mathrm{CI} 1.64-2.88, \chi^{2}=55.54, p=0.000\right)$. BMI was lower in patients with major depressive disorder (44.9 \pm 7.89$)$ than in subjects without mood disorders $\left(46.75 \pm 7.99, p_{\text {adj }}=0.017\right)$.

Conclusion Bariatric patients show high rates of psychiatric disorders, especially binge eating and mood disorders. Longitudinal studies are needed to explore the possible influence of such comorbidities on the long-term outcome after bariatric surgery. Level of evidence $\mathrm{V}$, cross sectional descriptive study.
\end{abstract}

Keywords Mood disorders · Panic disorder · Eating disorders · Obesity $\cdot$ Bariatric surgery

\section{Introduction}

Obesity, defined by a body mass index $\left(B M I=\mathrm{kg} / \mathrm{m}^{2}\right)$ of 30 or greater, is a chronic disease with a multifactorial etiopathogenesis, involving genetics, environment, metabolism, lifestyle and behavioral components. In recent decades, obesity has reached pandemic proportions, especially in Western countries. Excess weight is one of the most common risk

Giulio Perugi

giulio.perugi@med.unipi.it

1 Psychiatry 2 Unit, Department of Clinical and Experimental Medicine, University Hospital of Pisa, Via Savi 10, 56126 Pisa, Italia

2 Endocrinology Unit, Department of Clinical and Experimental Medicine, Obesity and Lipodystrophy Research Center, University Hospital of Pisa, Pisa, Italy factors underlying major chronic diseases such as heart disease, stroke, cancer, chronic respiratory disease and diabetes, which are the leading cause of mortality worldwide [1].

At present, bariatric surgery is the most durable weightloss treatment for severe obesity. It is recommended in patients with a BMI greater than $40 \mathrm{~kg} / \mathrm{m}^{2}$, or in those with a lower BMI if obesity-related comorbidities, such as type 2 diabetes and cardiovascular diseases, are present. Surgical treatment for obesity results in greater weight loss than conservative treatments, such as lifestyle interventions and pharmacological therapies, and is associated with significant improvement in obesity-related comorbidities and reduced mortality rate [2].

Psychiatric assessment is widely recommended during the multidisciplinary evaluation performed prior to bariatric surgery. In fact, psychiatric disorders are thought to have an impact on various post-surgical outcomes, both in 
the short- and the long-term, including weight loss, mental health and quality of life [3, 4]. However, at present, most authors believe that psychiatric comorbidity does not contraindicate surgery, with a few exceptions such as current substance abuse [5].

Although no conclusive data have been produced, there is strong evidence of a close bidirectional relationship between obesity and various psychiatric disorders [5-7]. This association appears to strengthen with increasing BMI and to be moderated by several variables, such as age, gender, or socioeconomic status $[8,9]$. In addition, bariatric patients seem to exhibit significantly higher rates of psychopathology than obese individuals who do not seek treatment or obese individuals who adhere to conservative programs that emphasize dietary restriction or promote weight control [4, 10].

Binge eating, mood and anxiety disorders are the most often detected conditions in obese bariatric patients [11]. Although binge-eating disorder (BED) is not limited to obese individuals, it is most common in this group and is often associated with various forms of psychopathology, including mood and anxiety symptoms [12]. Among mood disorders, depression is the most investigated condition in obese individuals seeking weight-loss treatment, whereas bipolar disorder is often overlooked. The latter is a chronic recurrent disorder characterized by abnormal fluctuations in mood state and energy. Bipolar type I and II disorders differ from unipolar depressive disorder since patients also exhibit pronounced episodes of mood elevation (manic and hypomanic episodes, respectively) [13]. Finally, among anxiety disorders, most previous reports on bariatric patients have focused on panic disorder. This psychiatric condition is characterized by recurrent panic attacks, which are sudden, intense bursts of anxiety or fear accompanied by a range of physical symptoms and maladaptive behaviors [14].

There are few comprehensive studies investigating psychiatric comorbidity through structured interviews in large populations of pre-surgical obese patients. Therefore, the aim of the present research was to provide lifetime prevalence rates of mood, eating, and panic disorders in a very large Italian sample of bariatric patients. A secondary objective was to explore patterns of psychiatric comorbidity and their relationship with BMI.

\section{Patients and methods}

\section{Patient sample and data collection}

The sample of this cross sectional and observational study was composed of adult ( $\geq 18$ years) obese bariatric patients. Without any exclusion criteria, we included in the study sample all subjects consecutively referred for bariatric surgery to the Obesity Center of the Endocrinology Unit in Pisa University Hospital, between January 2004 and November 2016. All patients were routinely interviewed by licensed psychiatrist during the pre-surgical evaluation.

Clinical charts were retrieved and examined. For any given patient, two independent researchers retrieved the data and filled a pre-defined data abstraction form. Any disagreement was resolved by consensus after discussion. All the medical records available were searched for sociodemographic information, anthropometric variables, i.e., weight and BMI, and lifetime diagnoses of mood, anxiety and eating disorders. Particularly, the diagnoses of major depressive disorder, bipolar disorders, panic disorder, BED, bulimia and anorexia nervosa were registered. To better define psychiatric diagnoses, the Structured Clinical Interview for DSM-IV Axis I disorders (SCID-I) [15] was used during the clinical interviews conducted over the years by trained psychiatrists.

\section{Statistical analysis}

Descriptive statistics were used to summarize the characteristics of the sample. The prevalence of mood, panic and eating disorders were computed and confidence intervals with 95\% confidence level $(95 \% \mathrm{CI})$ were obtained according to Clopper and Pearson [16]. Pearson's chi-squared tests were used to identify: (1) pairwise associations between mood disorders, panic disorder and eating disorders; (2) associations between major depressive vs. bipolar disorders and panic or eating disorders, within patients affected by mood disorders; (3) differences in gender proportions among all psychiatric comorbidity groups. A post-hoc analysis based on residuals was applied to the latter test, using the Benjamini-Hochberg method to adjust the significance level for multiple comparisons. Analyses of Variance (ANOVA) were conducted to assess significant differences of age and BMI between psychiatric comorbidity groups, the latter analysis including effects of age and gender. A Tukey's post-hoc test was used whenever the ANOVA led to a statistically significant result to retrieve significant comparisons between variables. A statistical significance level of $p<0.05$ was set for all tests. All the analyses were performed using $\mathrm{R}$ Statistical Software (Foundation for Statistical Computing, Vienna, Austria).

\section{Results}

Our sample was composed of 871 obese patients referred for bariatric surgery. The female gender accounted for $72 \%$ of the sample and age ranged between 18 and 69 years, with a mean of approximately 45 years (Table 1 ). Weight ranged between a minimum of $76 \mathrm{~kg}$ to a maximum of $264 \mathrm{~kg}$, with a mean of $126.48 \pm 26.07 \mathrm{~kg}$. BMI 
Table 1 Sociodemographic characteristics of obese patients referred to bariatric surgery $(N=871)$

\begin{tabular}{lc}
\hline Female gender $(n, \%)$ & $629(72.2)$ \\
Age, years (mean, SD) & $45.36(10.84)$ \\
BMI, kg/m ${ }^{2}$ (mean, SD) & $46.32(7.85)$ \\
Marital status $(n, \%)$ & \\
Never married & $131(19.3)$ \\
Married & $470(69.2)$ \\
Divorced & $62(9.1)$ \\
Widowed & $16(2.4)$ \\
Education level $(n, \%)$ & \\
Degree & $68(10.2)$ \\
High school diploma & $262(39.1)$ \\
Middle school diploma & $273(40.8)$ \\
Primary school diploma & $67(10.0)$ \\
Employment status & \\
Employed & $509(65.3)$ \\
Unemployed & $192(24.6)$ \\
Retired & $57(7.3)$ \\
Student & $22(2.8)$ \\
\hline
\end{tabular}

$B M I$ body mass index

ranged between $31 \mathrm{~kg} / \mathrm{m}^{2}$ and $87 \mathrm{~kg} / \mathrm{m}^{2}$, with a mean of $46.32 \pm 7.85 \mathrm{~kg} / \mathrm{m}^{2}$. As expected, the majority of patients was diagnosed with class III obesity (BMI $\left.\geq 40 \mathrm{~kg} / \mathrm{m}^{2}\right)$, with more than three fourths of patients affected $(N=707$, $81.2 \%)$. A lower number of patients was diagnosed with class II obesity $\left(35 \mathrm{~kg} / \mathrm{m}^{2} \leq \mathrm{BMI}<40 \mathrm{~kg} / \mathrm{m}^{2}\right)(N=139$, $16.0 \%)$, while an almost negligible group of subjects was affected by class I obesity $\left(\right.$ BMI $\left.<35 \mathrm{~kg} / \mathrm{m}^{2}\right)(N=25$, $2.8 \%$ ). Marital status was available for 679 patients, educational level for 670 patients, and employment status for 780 subjects. More than half of the patients were married/ cohabiting (69\%) and employed $(65 \%)$ at the time of the evaluation; the vast majority of the sample had a middle or high school diploma (respectively, $41 \%$ and 39\%).

Mood and eating disorders were highly prevalent in the sample (Table 2). Lifetime major depressive disorders or bipolar disorders were diagnosed in 338 patients (38.7\%); BED, bulimia nervosa and anorexia nervosa were diagnosed in 259 subjects $(29.7 \%)$. BED was the most prevalent condition, involving most of the patients with eating disorders (93.1\%). One of three subjects with anorexia nervosa and more than a half of patients with bulimia nervosa $(N=19,54.3 \%)$ were indeed also diagnosed with BED. Among mood disorders, major depressive disorder and bipolar disorder type 2 were the most frequently diagnosed, followed, in order, by cyclothymia/ other specified bipolar disorder and bipolar disorder type 1. However, bipolar disorders represented the second most frequent diagnosis overall $(N=198,22.7 \%, 95 \%$ $\mathrm{CI}=20.0-25.7)$, exceeding both major depressive disorder
Table 2 Lifetime prevalence of mood, panic and eating disorders in obese patients referred to bariatric surgery $(N=871)$

\begin{tabular}{lrlc}
\hline & $N$ & $\%$ & $95 \%$ CI \\
\hline Any psychiatric disorder & 480 & $55.1 \%$ & $51.7-58.5$ \\
Any affective disorder & 338 & 38.7 & $35.5-42.0$ \\
Major depressive disorder & 140 & 16.0 & $13.7-18.6$ \\
Bipolar disorder type 1 & 21 & 2.4 & $1.5-3.7$ \\
Bipolar disorder type 2 & 135 & 15.5 & $13.1-18$ \\
Cyclothymia/other specified & 42 & 4.8 & $3.5-6.4$ \\
$\quad$ bipolar disorder & & & \\
Any eating disorder & 259 & $29.7 \%$ & $26.7-32.9$ \\
Binge-eating disorder & 241 & 27.6 & $24.7-30.7$ \\
Bulimia nervosa & 35 & 4.0 & $2.8-5.5$ \\
$\quad$ Anorexia nervosa & 3 & 0.3 & $0.1-1$ \\
Panic disorder & 140 & 16.0 & $13.7-18.6$ \\
\hline
\end{tabular}

and panic disorder. The latter two conditions showed the same prevalence.

Significant pairwise associations between mood, panic and eating disorders were found in the whole sample $(N=871)$ (Fig. 1). Mood disorders showed the strongest association with panic disorder (OR 2.75, 95\% CI 1.90-3.99, $\left.\chi^{2}=41.85, p=0.000\right)$. In addition, twice as many patients were diagnosed with eating disorders among mood disordered subjects with respect to unaffected individuals $\left(\mathrm{OR}=2.17,95 \% \mathrm{CI}=1.64-2.88, \chi^{2}=55.54, p=0.000\right)$. Also eating disorders and panic disorder were significantly associated $\left(\mathrm{OR}=1.99,95 \% \mathrm{CI}=1.38-2.86, \chi^{2}=19.49\right.$, $p=0.000$ ), with most of comorbid patients also showing a mood comorbidity (46 of $64,71.9 \%$.

Within mood disordered patients $(N=338)$, no significant association between bipolar disorders and eating disorders was observed, with 56 of major depressive patients (40.0\%) and 94 of bipolar patients (47.5\%) being comorbid $\left(\chi^{2}=1.57, p=0.211\right)$. Conversely, a significant association between major depressive disorder and panic disorder comorbidity was found within mood disordered patients $\left(N=338, \chi^{2}=4.69, p=0.030\right)$. Indeed, 46 of major depressive patients $(32.9 \%)$ were diagnosed with panic disorder, compared to 43 of patients with bipolar disorder (21.7\%). Multimorbid patients with mood, panic and eating disorders $(N=46)$, were equally represented in both the groups with major depressive disorder $(N=23,16.4 \%)$ and bipolar disorders $(N=23,11.6 \%)\left(N=338, \chi^{2}=1.23, p=0.267\right)$.

Gender proportions were found to significantly differ between diagnostic groups $\left(\chi^{2}=43.78, \mathrm{df}=11, p=0.000\right)$. Males were overrepresented in subjects without psychiatric comorbidity (143 of 391, 36.6\%) as compared to all the other patients diagnosed with at least one among mood, panic and eating disorders (99 of 480 overall, 20.6\%), as suggested by post-hoc residuals analysis (residual $=5.23$, 
Fig. 1 Patterns of comorbidity between mood, panic and eating disorders in obese patients referred to bariatric surgery $(N=871)$. " $N$ " is used for three group sample size, while " $n$ " indicates subgroup sample size

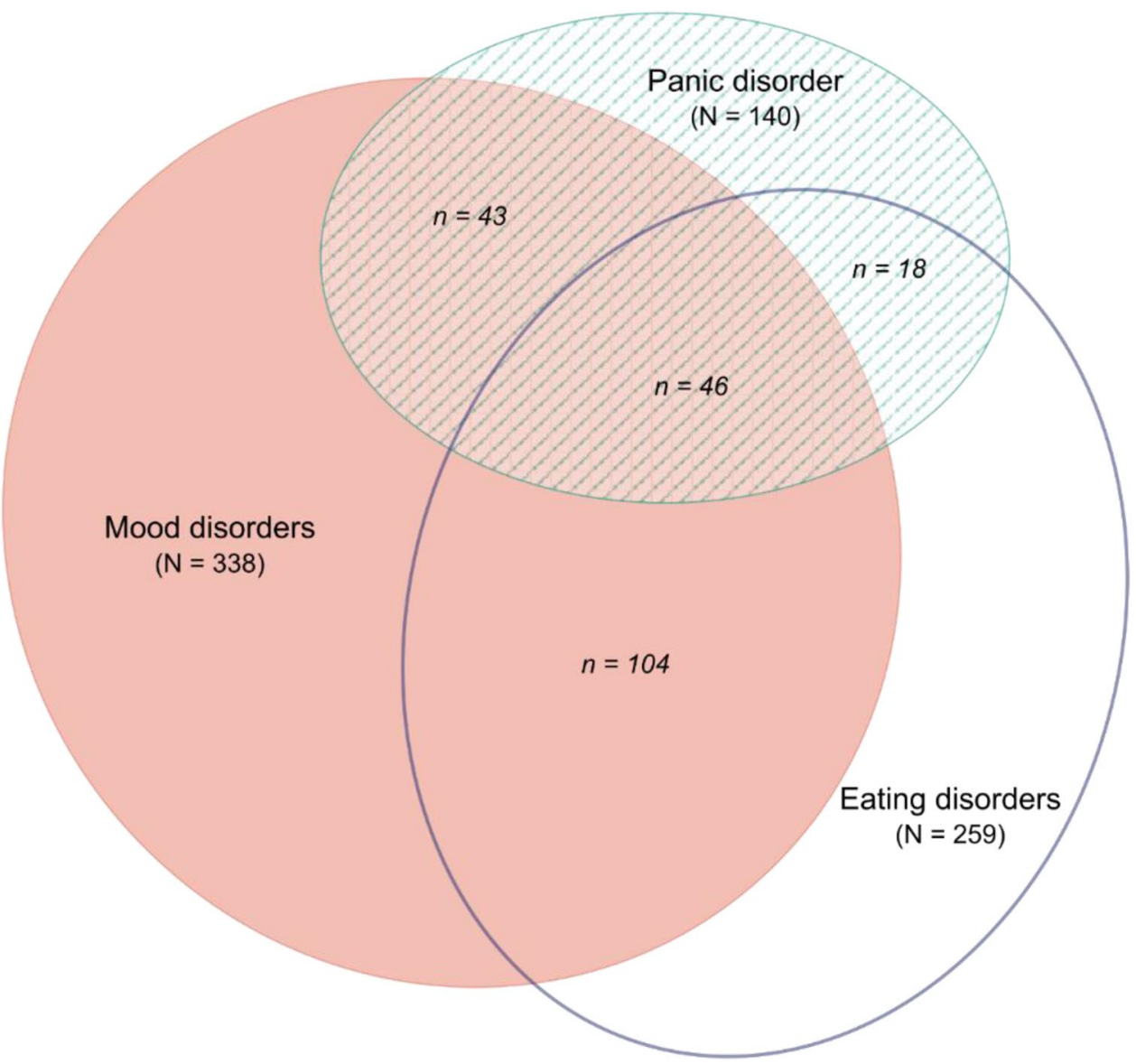

$\left.p_{\text {adj }}=0.000\right)$. As for age, a significant effect of mood disorders $[\mathrm{F}(2,859)=6.14, p=0.002]$ and a significant interaction between the effects of mood disorders and eating disorders $[F(2,859)=3.40, p=0.034]$ on age emerged. Post-hoc contrasts revealed that patients with major depressive disorder $(N=140$, age $=47.63 \pm 10.79)$ were significantly older than patients without mood disorders $(N=533$, age $\left.=44.95 \pm 10.98, p_{\text {adj }}=0.002\right)$. In addition, patients with major depressive disorder without eating disorders $(N=84$, age $=50.61 \pm 10.24)$ were significantly older than patients without both the disorders $(N=424$, age $=45.36 \pm 11.05$, $\left.p_{\text {adj }}=0.001\right)$, with eating disorders only $(N=109$, age $\left.=43.35 \pm 10.57, p_{\text {adj }}=0.000\right)$, with major depressive disorder and eating disorders $(N=56$, age $=43.16 \pm 10.11$, $\left.p_{\text {adj }}=0.001\right)$, and with bipolar and eating disorders $(N=94$, age $=42.5 \pm 9.92, p_{\text {adj }}=0.000$ ). Finally, patients with bipolar and eating disorders were significantly younger than patients with bipolar disorders only $(N=104$, age $=46.99 \pm 10.28$, $p_{\text {adj }}=0.035$ ).

Only a significant effect of mood disorders on BMI was observed $[\mathrm{F}(2,825)=4.29, p=0.014]$. Post-hoc contrasts revealed that patients with major depressive disorder $(n=140, \mathrm{BMI}=44.9 \pm 7.89)$ showed, on average, a significantly lower BMI than patients without mood disorders $\left(n=533, \mathrm{BMI}=46.75 \pm 7.99, p_{\text {adj }}=0.017\right)$.

\section{Discussion}

This is, to our knowledge, the largest study $(n=871)$ exploring the prevalence of psychiatric disorders through the administration of a structured interview in a population of obese patients seeking for weight-loss surgical treatment. In particular, we investigated the prevalence of mood, panic and eating disorders, according to DSM-IV criteria. The psychiatric assessment was performed by trained psychiatrists and took place during the multidisciplinary pre-surgical evaluation of the patients.

Similarly to most of the previous reports [10, 17-25], almost three-quarters of the sample were women, the mean age was 45 years, and the vast majority of patients belonged to class III obesity. Overall, the sample showed a lower level of education than the general population in Italy [26]. In accordance with the dramatic increase over the years of the most severe forms of obesity [27], in the current sample, we found a higher rate of class III obesity $(80 \%)$ than in a 
previous sample of bariatric patients recruited at the University Hospital of Pisa (66\%) [28].

Consistently with the growing evidence of a particularly high rate of psychiatric comorbidities in obese patients referred to bariatric surgery, in the current sample, $55 \%$ of participants were diagnosed with at least one lifetime psychiatric disorders. Men showed a lower rates of any lifetime psychiatric disorder than women, in agreement with some previous reports $[18,24,28]$. However, gender differences may reflect a male tendency to minimize psychiatric conditions and to report them less frequently to the physician [29].

To date, there is still little clarity on the exact prevalence of psychiatric disorders in obese patients and, in particular, in bariatric subjects. The few studies addressing this issue often present small sample sizes and use heterogeneous methodologies, such as different interviewing strategies (e.g., in-person versus telephone-interview) and psychometric questionnaires. In addition, accurate estimates are difficult to obtain because of the tendency of bariatric patients to underreport psychiatric conditions to access surgery [4]. As a consequence, a wide range of lifetime psychiatric disorders rates were reported by different studies (37-81\%), both in USA and in Europe [10, 19, 28, 30-32].

In our sample, the most frequent lifetime psychiatric conditions were mood disorders, affecting approximately $39 \%$ of the sample. Specifically, $16 \%$ of patients were diagnosed with major depressive disorder and 23\% with bipolar spectrum disorders, among which bipolar disorder type II was the most represented (15.5\%). In agreement with previous reports [17, 20,25], patients with mood disorders showed higher rates of comorbidity with eating and panic disorders than other patients. Unexpectedly, panic disorder was found to be significantly associated with major depression but not bipolar disorder.

In previous studies assessing psychiatric comorbidity in bariatric subjects, the prevalence of major depressive disorder and bipolar disorder type II were more prone to vary, while bipolar disorder type I showed an almost stable prevalence of $1-5 \%$ [24]. Considering the entire spectrum of bipolar disorders, some authors have reported very low rates $(1-6 \%)[10,20,21,28]$ while others have found higher percentages (33-37\%) [19, 25, 31]. In an Italian sample, Alciati et al. [33] observed an impressive prevalence of broad bipolar spectrum disorders (89\%), according to Angst's classification [34]. Finally, only four previous studies have found comparable or higher rates of bipolar spectrum disorders than depressive disorders in samples of bariatric subjects $[19,25,31,33]$. The extreme variability in the prevalence of mood disorders probably stems from different operational criteria being used in previous studies. In addition, bipolar disorder type II is frequently underdiagnosed or misdiagnosed with major depressive disorder, due to the difficult retrospective assessment of hypomanic episodes [35]. Importantly, in two large samples of patients with a major depressive episode, the presence of obesity was associated with higher rates of bipolar spectrum disorders and (hypo)manic symptoms, suggesting that obesity could be considered a marker of bipolarity in major depressive patients [36, 37]. Consistently with our study, bipolarity features were observed in approximately one half of depressed obese patients [36].

High variability also exists in literature regarding the percentage of bariatric patients showing a comorbidity with BED (5-49\%) [10, 18, 19, 21, 28, 30, 31] and panic disorder $(1-30 \%)[10,19,28,32]$. In the current report, BED was detected in almost one third of the entire sample and represented the most frequent individual diagnosis. Obese patients with comorbid BED have been previously shown to exhibit significantly higher rates psychiatric disorders compared to obese patients without BED, in particular among bariatric surgery candidates [18, 23, 24, 28]. Overall, recent evidence suggests that the presence of an eating disorder may be a marker for other psychiatric conditions, primarily mood and anxiety disorders [38]. A common diathesis of emotional dysregulation and impulsivity, predisposing to compulsive and addictive behaviors, was proposed as a mediating mechanism between BED, obesity, and bipolar disorders [25].

Finally, in our sample, BMI was found to be lower in patients with major depressive disorder compared to subjects without mood disorders. In a previous study, Kalarchian et al. [21] have found higher BMI in subjects with comorbid psychiatric disorders compared to other patients, whereas Segura-Garcia et al. [17] found a lower BMI in subjects with bipolar spectrum disorder compared to the others. However, most studies have failed to find clear associations between BMI and particular psychiatric conditions [20, 23, 25, 28] and more homogeneous future studies addressing this issue are certainly needed.

\section{Conclusions}

Given the increasing prevalence of severe obesity and the rise of bariatric surgery worldwide, the evaluation of psychiatric disorders in bariatric candidates appears to play an increasingly important role. While not representing absolute contraindications to surgery, various psychiatric conditions could impact the long-term outcome after the intervention, both in terms of weight loss and quality of life. Identifying and treating mental disorders could improve patients' behaviors before and after surgery with important clinical and therapeutic implications. An integrated model of care should be used to ensure individualized treatments aligned with patient needs and symptom severity. Psychological interventions (primarily, cognitive and behavioral therapy) 
and psychopharmacological treatment have demonstrated to impact on disordered/maladaptive eating behaviors, psychological distress, and quality of life and should be considered in patients who are at risk for weight regain and adverse psychosocial outcomes [39].

\section{Strengths and limitations}

In our opinion, the major limitation of this study is that we were only able to explore a small group of psychiatric disorders because of the absence of a systematic evaluation of other diagnostic categories, such as neurodevelopmental disorders, that only recently received sufficient attention from researchers and clinicians. In addition, the psychiatric evaluation in this study was part of the pre-surgical assessment, which may have led patients to underestimate some aspects of their psychiatric status or history to access surgery. Further studies are needed in which the assessment process is strictly independent of the surgery approval process to better detect the presence of an underlying psychopathology. Another limitation is the absence of a control group of overweight patients, which would have been useful to unravel other differences in BMI among groups of patients. Finally, we were only able to provide data regarding lifetime psychiatric comorbidities, without indicating the prevalence of current disorders or the percentages of patients on psychopharmacological therapy at the time of assessment. On the other hand, the major strengths of our study are certainly the very large sample and the use of a structured diagnostic interview by trained psychiatrists to confirm psychiatric diagnoses.

\section{What is already known on this subject?}

Variably high lifetime prevalence rates of several mental disorders have been reported in relatively small samples of obese bariatric patients.

\section{What this study adds?}

This is, to our knowledge, the largest study confirming the high prevalence of psychiatric disorders, particularly mood disorders and binge eating disorder, in patients seeking bariatric surgery.

Funding Open access funding provided by Università di Pisa within the CRUI-CARE Agreement.

Availability of data and material Data are available on reasonable request from the corresponding author.

\section{Declarations}

Conflict of interest Prof. Perugi acted as consultant to Lundbeck, Angelini, FB-Health. He received a scholarship/research support from Lundbeck and Angelini. He is a member of the speaker/advisory board of Sanofi-Aventis, Lundbeck, FB-Health, Angelini. Prof. Santini has acted as a consultant, has received grant/research support and/or is on the speaker/advisory board for NovoNordisk, Bruno Farmaceutici, Aegerion-Amrit, Bio Italia srl. Prof Maremmani served as Board Member for Angelini, Camurus, CT Sanremo, D\&A Pharma, Gilead, Indivior, Lundbeck, Molteni, MSD, Mundipharma. Other authors have no affiliation or financial interest in any organization that may constitute a conflict of interest.

Ethical approval The study was carried out in accordance with The Code of Ethics of the World Medical Association (Declaration of Helsinki) and was approved by the Ethics Committee of the University of Pisa.

Consent to participate Because the study is based on retrieved clinical charts, informed consent is not required for participation in the study.

Consent for publication Because the study is based on retrieved clinical charts, informed consent is not required for publication.

Open Access This article is licensed under a Creative Commons Attribution 4.0 International License, which permits use, sharing, adaptation, distribution and reproduction in any medium or format, as long as you give appropriate credit to the original author(s) and the source, provide a link to the Creative Commons licence, and indicate if changes were made. The images or other third party material in this article are included in the article's Creative Commons licence, unless indicated otherwise in a credit line to the material. If material is not included in the article's Creative Commons licence and your intended use is not permitted by statutory regulation or exceeds the permitted use, you will need to obtain permission directly from the copyright holder. To view a copy of this licence, visit http://creativecommons.org/licenses/by/4.0/.

\section{References}

1. Heymsfield SB, Wadden TA (2017) Mechanisms, pathophysiology, and management of obesity. N Engl J Med 376:254-266. https://doi.org/10.1056/NEJMra1514009

2. Colquitt JL, Pickett K, Loveman E, Frampton GK (2014) Surgery for weight loss in adults. Cochrane Database Syst Rev. https://doi. org/10.1002/14651858.CD003641.pub4

3. Wimmelmann CL, Dela F, Mortensen EL (2014) Psychological predictors of weight loss after bariatric surgery: a review of the recent research. Obes Res Clin Pract 8:e299-313. https://doi.org/ 10.1016/j.orcp.2013.09.003

4. Malik S, Mitchell JE, Engel S et al (2014) Psychopathology in bariatric surgery candidates: a review of studies using structured diagnostic interviews. Compr Psychiatry 55:248-259. https://doi. org/10.1016/j.comppsych.2013.08.021

5. Simon GE, Von Korff M, Saunders K et al (2006) association between obesity and psychiatric disorders in the US adult population. Arch Gen Psychiatry 63:824. https://doi.org/10.1001/archp syc.63.7.824

6. Rajan TM, Menon V (2017) Psychiatric disorders and obesity: a review of association studies. J Postgrad Med 63:182-190. https:// doi.org/10.4103/jpgm.JPGM_712_16 
7. McElroy SL, Kotwal R, Malhotra S et al (2004) Are mood disorders and obesity related? A review for the mental health professional. J Clin Psychiatry 65:634-651. https://doi.org/10.4088/jcp. v65n0507

8. Tronieri JS, Wurst CMC, Pearl RL, Allison KC (2017) Sex differences in obesity and mental health. Curr Psychiatry Rep 19:29. https://doi.org/10.1007/s11920-017-0784-8

9. Weiss F, Barbuti M, Carignani G et al (2020) Psychiatric aspects of obesity: a narrative review of pathophysiology and psychopathology. J Clin Med 9:2344. https://doi.org/10.3390/jcm9082344

10. Lin HY, Huang CK, Tai CM et al (2013) Psychiatric disorders of patients seeking obesity treatment. BMC Psychiatry 13:1. https:// doi.org/10.1186/1471-244X-13-1

11. Dawes AJ, Maggard-Gibbons M, Maher AR et al (2016) Mental health conditions among patients seeking and undergoing bariatric surgery a meta-analysis. JAMA - J Am Med Assoc 315:150-163. https://doi.org/10.1001/jama.2015.18118

12. Mitchell JE, Devlin MJ, De Zwann M et al (2007) Binge-eating disorder: clinical foundations and treatment. The Guilford Press, New York

13. Grande I, Berk M, Birmaher B, Vieta E (2016) Bipolar disorder. Lancet 387:1561-1572. https://doi.org/10.1016/S0140-6736(15) 00241-X

14. Roy-Byrne PP, Craske MG, Stein MB (2006) Panic disorder. Lancet 368:1023-1032. https://doi.org/10.1016/S0140-6736(06) 69418-X

15. First MB, Spitzer RL, Gibbon M, Williams JB (1996) Structured clinical interview for DSM-IV axis I disorders. American Psychiatric Publishing, Arlington, VA

16. Clopper CJ, Pearson ES (1934) The use of confidence or fiducial limits illustrated in the case of the binomial. Biometrika 26:404413. https://doi.org/10.2307/2331986

17. Segura-Garcia C, Caroleo M, Rania M et al (2017) Binge Eating Disorder and Bipolar Spectrum disorders in obesity: psychopathological and eating behaviors differences according to comorbidities. J Affect Disord 208:424-430. https://doi.org/10.1016/j.jad. 2016.11.005

18. Rosenberger PH, Henderson KE, Grilo CM (2006) Psychiatric disorder comorbidity and association with eating disorders in bariatric surgery patients: a cross sectional study using structured interview-based diagnosis. J Clin Psychiatry 67:1080-1085. https://doi.org/10.4088/JCP.v67n0710

19. Duarte-Guerra LS, Coêlho BM, Santo MA, Wang YP (2015) Psychiatric disorders among obese patients seeking bariatric surgery: results of structured clinical interviews. Obes Surg 25:830-837. https://doi.org/10.1007/s11695-014-1464-y

20. Grothe KB, Mundi MS, Himes SM et al (2014) Bipolar disorder symptoms in patients seeking bariatric surgery. Obes Surg 24:1909-1914. https://doi.org/10.1007/s11695-014-1262-6

21. Kalarchian MA, Marcus MD, Levine MD et al (2007) Psychiatric disorders among bariatric surgery candidates: relationship to obesity and functional health status. Am J Psychiatry 164:328-334. https://doi.org/10.1176/ajp.2007.164.2.328

22. Benzerouk F, Gierski F, Ducluzeau PH et al (2018) Food addiction, in obese patients seeking bariatric surgery, is associated with higher prevalence of current mood and anxiety disorders and past mood disorders. Psychiatry Res 267:473-479. https://doi.org/10. 1016/j.psychres.2018.05.087

23. Jones-Corneille LR, Wadden TA, Sarwer DB et al (2012) Axis i psychopathology in bariatric surgery candidates with and without binge eating disorder: results of structured clinical interviews. Obes Surg 22:389-397. https://doi.org/10.1007/ s11695-010-0322-9

24. Mühlhans B, Horbach T, de Zwaan M (2009) Psychiatric disorders in bariatric surgery candidates: a review of the literature and results of a German pre-bariatric surgery sample. Gen Hosp Psychiatry 31:21. https://doi.org/10.1016/j.genhosppsych.2009. 05.004

25. Barbuti M, Carignani G, Weiss F et al (2021) Mood disorders comorbidity in obese bariatric patients: the role of the emotional dysregulation. J Affect Disord 279:46-52. https://doi.org/10. 1016/j.jad.2020.09.128

26. ISTAT (2019) Livelli di istruzione e ritorni occupazionali. https:// www.istat.it/it/files/2019/07/Report-Livelli-di-istruzione-e-ritor ni-occupazionali_2018.pdf

27. Sturm R, Hattori A (2013) Morbid obesity rates continue to rise rapidly in the United States. Int J Obes 37:889-891. https://doi. org/10.1038/ijo.2012.159

28. Mauri M, Rucci P, Calderone A et al (2008) Axis I and II disorders and quality of life in bariatric surgery candidates. J Clin Psychiatry 69:295-301. https://doi.org/10.4088/JCP.v69n0216

29. World Health Organization (WHO). (2002) Gender and Mental Health

30. Mitchell JE, Selzer F, Kalarchian MA et al (2012) Psychopathology before surgery in the Longitudinal Assessment of Bariatric Surgery-3 (LABS-3) psychosocial study. Surg Obes Relat Dis 8:533-541. https://doi.org/10.1016/j.soard.2012.07.001

31. Borgès Da Silva V, Borgès Da Silva R, Prud'homme A et al (2018) Association between binge eating disorder and psychiatric comorbidity profiles in patients with obesity seeking bariatric surgery. Compr Psychiatry 87:79-83. https://doi.org/10.1016/j.comppsych. 2018.09.004

32. Barbuti M, Mazzarini L, Vieta E et al (2019) Relationships between recurrence and polarity in major depressive disorders: pooled analysis of the BRIDGE and BRIDGE-II-MIX cohorts. J Affect Disord 256:250-258. https://doi.org/10.1016/j.jad.2019. 06.005

33. Alciati A, D'Ambrosio A, Foschi D et al (2007) Bipolar spectrum disorders in severely obese patients seeking surgical treatment. J Affect Disord 101:131-138. https://doi.org/10.1016/j.jad.2006.11. 008

34. Angst J, Gamma A, Benazzi F et al (2003) Toward a re-definition of subthreshold bipolarity: epidemiology and proposed criteria for bipolar-II, minor bipolar disorders and hypomania. J Affect Disord 73:133-146

35. Da Silva VB, Da Silva RB, Azorin JM, Belzeaux R (2015) Mood disorders are highly prevalent but underdiagnosed among patients seeking bariatric surgery. Obes Surg 25:4. https://doi.org/10.1007/ s11695-014-1557-7

36. Petri E, Bacci O, Barbuti $\mathrm{M}$ et al (2017) Obesity in patients with major depression is related to bipolarity and mixed features: evidence from the BRIDGE-II-Mix study. Bipolar Disord $19: 458-464$

37. Vannucchi G, Toni C, Maremmani I, Perugi G (2014) Does obesity predict bipolarity in major depressive patients? J Affect Disord 155:118-122. https://doi.org/10.1016/j.jad.2013.10.035

38. Stunkard AJ, Allison KC (2003) Binge eating disorder: disorder or marker? Int J Eat Disord 34:S107-S116. https://doi.org/10.1002/ eat. 10210

39. Sockalingam S, Leung SE, Wnuk S et al (2020) Psychiatric management of bariatric surgery patients: a review of psychopharmacological and psychological treatments and their impact on postoperative mental health and weight outcomes. Psychosomatics 61:498-507. https://doi.org/10.1016/j.psym.2020.04.011

Publisher's Note Springer Nature remains neutral with regard to jurisdictional claims in published maps and institutional affiliations. 